\title{
AUTOMATIC NUMBER PLATE RECOGNITION FOR DIFFERENT FONTS AND NON-ROMAN SCRIPT
}

\author{
Kirad Varad Vinay \\ Department of Computer \\ Engineering \\ JSPM's Jayawantrao Sawant \\ College of Engineering, Pune, \\ Maharashtra, India
}

\author{
Indla Omkar Balaobaiah \\ Department of Computer \\ Engineering \\ JSPM's Jayawantrao Sawant \\ College of Engineering, Pune, \\ Maharashtra, India
}

\author{
Mujawar Sohail Mahiboob \\ Department of Computer \\ Engineering \\ JSPM's Jayawantrao Sawant \\ College of Engineering, Pune, \\ Maharashtra, India
}

\author{
Shinde Dinesh Nagnath \\ Department of Computer Engineering \\ JSPM's Jayawantrao Sawant College of Engineering, \\ Pune, Maharashtra, India
}

\author{
Prof. Darshana patil \\ Department of Computer Engineering \\ JSPM's Jayawantrao Sawant College of Engineering, \\ Pune, Maharashtra, India
}

\begin{abstract}
According to survey taken the total number of vehicles in [1] India were 260 million. Therefore, there is a need to develop Automatic Number Plate Recognition (ANPR) systems [1] in India because of the large number of vehicles travelling on the roads. [1] It would also help in proper tracking of the vehicles, traffic examining, finding stolen vehicles, supervising parking toll and imposing strict actions against red light breaching. Automatic number plate recognition is image processing technique for finding number plate from image and extracting characters from detected number plate. ANPR in India has always been challenging due to different lighting conditions, changes in fonts, shapes, angles, letters size, number of lines and padding between lines, different languages used. In our project we proposed a model that can detects number plate with considering all irregularities. this system uses Computer vision and machine learning technology in order to detect number plate from image. In our proposed system number plate can be of different fonts and non-roman script. For identification of characters from number plate we use OCR (Optical character recognition) technique. OCR involves two parts: Character segmentation and Character Recognition. This OCR system can be used to extract characters of different fonts and non-roman script. The Quality of OCR depends on the quality of image, image contrast, text font style and size. To improve quality of OCR we can use image processing technique to enhance quality of image.
\end{abstract}

Keywords - Recognition, ANPR, OCR (Optical character recognition), license plate, $\mathrm{CNN}$

\section{INTRODUCTION}

Every country has their own ANPR system for traffic control, traffic surveillance, vehicle monitoring system against illegal activities, security control in restricted areas, parking management, toll collection, traffic law enforcement and identification of unregistered vehicles. [2] and as such India also has its own system of assigning unique numbers to vehicles. These unique numbers are assigned to the vehicle by RTO (Regional Transport Office). These are generally easily readable by humans but when it comes to do the same by using machines many effects such as illumination, foreground color and blur background poses a problem, Hence Automatic number plate recognition can be used to detection of number plate.

Automatic number plate recognition finds the number plate from images using image processing and deep learning and create bounding box around the number plate. Automatic number plate recognition is used in Intelligent transport system and smart city.

ANPR system consists of mainly three parts, License plate detection, Character segmentation and character recognition. Each part can be trained with different images and different conditions. The problems with ANPR can be resolved using Machine learning technology with proper training with respect to vehicles images at different lighting conditions and different font styles. Number plate is detected from the input image and 


\section{International Journal of Engineering Applied Sciences and Technology, 2021 \\ Vol. 5, Issue 11, ISSN No. 2455-2143, Pages 314-317 \\ Published Online March 2021 in IJEAST (http://www.ijeast.com)}

it is used for character segmentation and character recognition. Detection of number plate from image is a difficult task due to different size of number plate, font styles. If the system fails to detect number plate from image it will affect the accuracy and further steps used. Convolutional Neural Network (CNN) is a powerful classification tool which can be used to various object detection task. CNN can be used in plate detection, character segmentation and recognition with improved accuracy. It can detect number plate from image without pre-segmentation. ANPR can be used to store the images of vehicle taken by cameras as well as the extracted text from the license plate. It also can be used for improving traffic flow. ANPR helps in enhancing security by assisting the traffic police to detect the rule breakers, overspeed vehicles and frauds on road.

\section{METhodology}

\section{A. Number plate detection-}

In India number plates are irregular in terms of shape and size. Every type vehicle is having number plates of different shape and size. So, the best way to identify the number plate from image is using pretrained deep learning model. In order to train deep learning model to identify the number plate from image we have to follow following steps.

- Preparing dataset

- Labelling Images

- Training

- Output

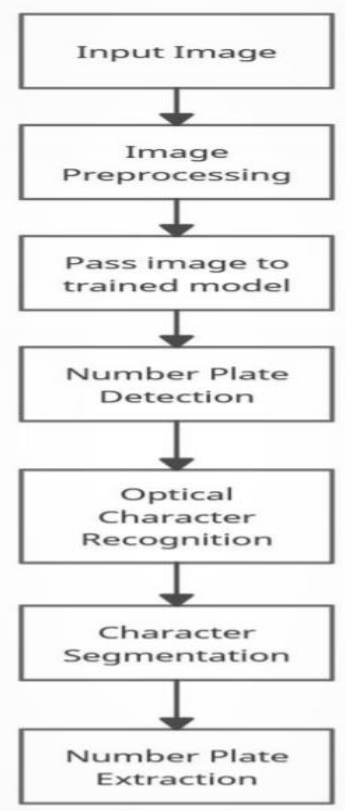

Fig. 1.Automatic number plate detection and OCR system

1. Preparing dataset
For Detection of number plate from image on of the most important factor is quality of dataset. If the quality of dataset is not good then it can lead to wrong prediction of number plate. So that we have to make sure that dataset contains all layouts of number plates possible. For this we created a dataset contain cars, two wheelers, mini trucks, auto rikshaw and lorries with different layouts of number plate.

The first step in data preparation is collecting images. As there are various types of vehicles and that contain number plate of different shapes and size. So, we have to collect images that contain different types of number plate and.

The second step in data preparation is splitting these images into Train and Test Folder. We have to split data for training and testing of deep learning model. We will put $80 \%$ of images in Train folder and $20 \%$ of images in Test folder.

\section{Labelling images}

The next step in number plate detection is labelling image dataset. Labelling dataset is the process of collecting raw data and labelling each feature from image so that machine learning model can learn from that data. Labelling is used to classify features form dataset. This labelled image dataset is used as training dataset for training deep learning model. In this we are creating bounding box to a number plate for further training process.

In order to label our data, we need image labeling software. We used LabelImg software to label Images.

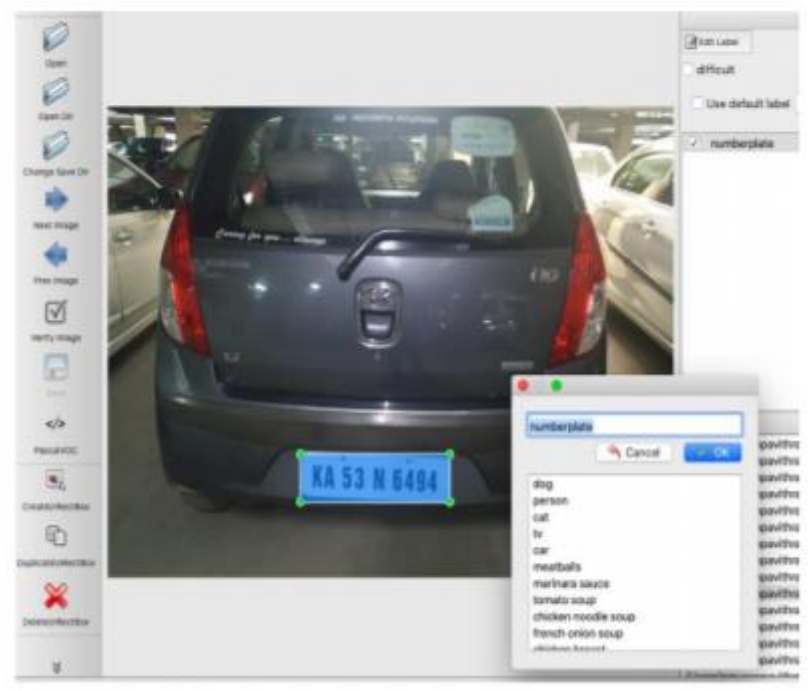

Fig. 2. Labeling number plate from image

\section{Training}

Deep learning is a machine learning technique that teaches computer what comes naturally to human: learn by example. In deep learning, a computer model learns to perform classification task on images. In this model is trained by using large set of labelled data and Convolutional neural network that 
contains many layers. Convolutional neural network is trained by optimizing on cross entropy loss function using mini batch gradient descent and black propagation.

\section{Output}

After training model using convolutional neural network $(\mathrm{CNN})$ this gives output containing bounding box to number plate. Fig. 3 shows output of trained model.

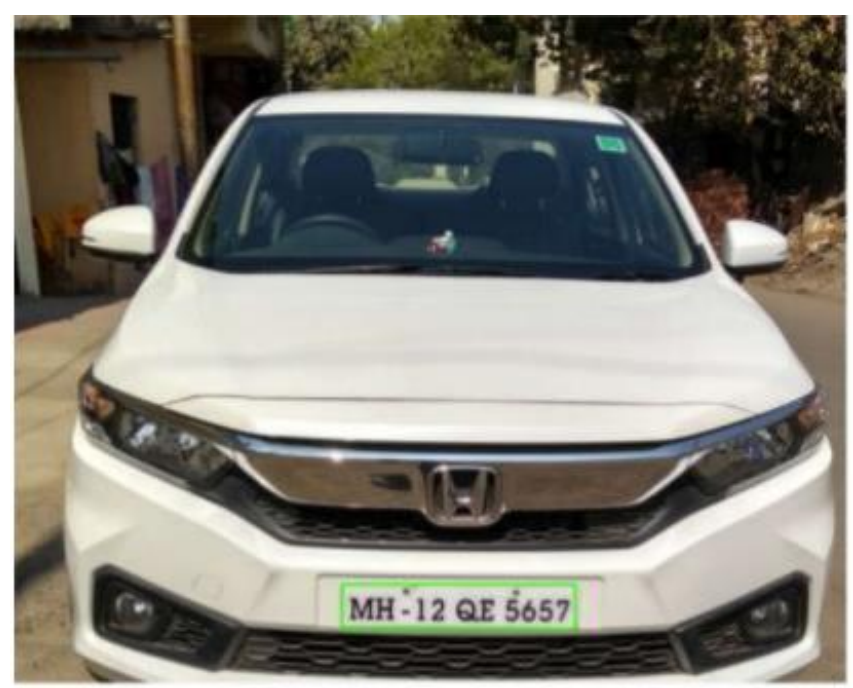

Fig 3. Output

\section{B. Optical character Recognition (OCR)-}

Optical character recognition contains following steps

- Character Segmentation

- Character Recognition

Algorithm to extract characters from number plate

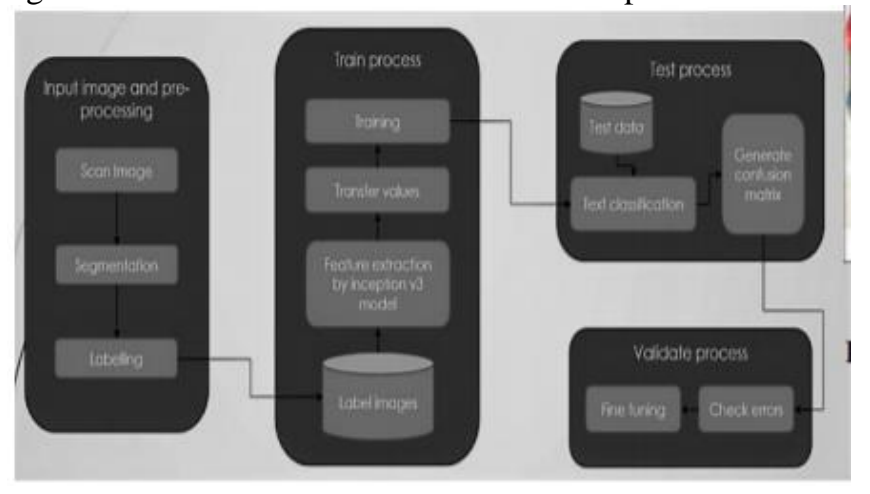

Fig 4. Algorithm for OCR

\section{Character segmentation}

After detection of number plate from image we have to perform character segmentation for character recognition. character segmentation is the process of separating each character from the number plate.
After completion of character segmentation, we get the bounding box on each character with its co-ordinates.

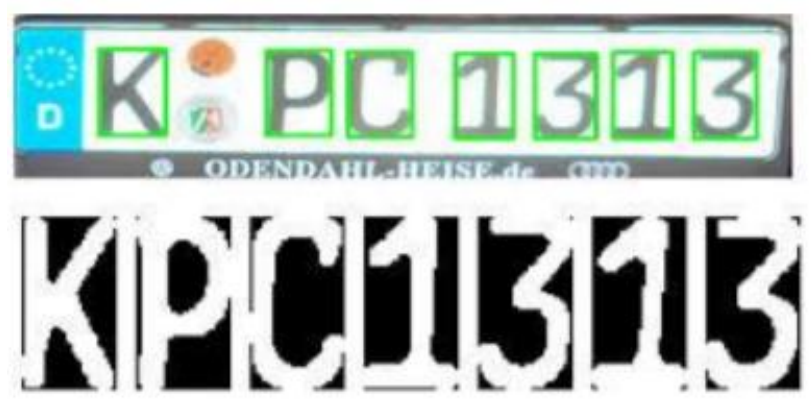

Fig 4. Character Segmentation

\section{Character recognition}

Character recognition finds the feature from each and every character from image. template matching is one of the character recognition technique. Template matching algorithm finds the similarities between input and output images.

The major problem with character recognition in number plate is the large number of font styles used in the plate. We found that the best way to recognize characters from image is to train neural network to recognize the numbers and characters from cropped image. In this way neural network can identify feature associated with each characters and number, and can also be used for recognizing various fonts including the painted number plates.

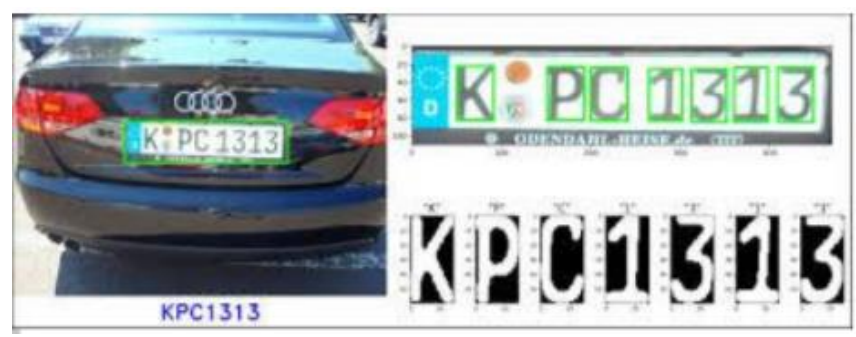

Fig 5. Character Recognition

\section{CONCLUSION}

As an overall view of the system it could be concluded that this system has been developed by using technique mentioned and elaborated earlier in this document which is Convolutional neural network $(\mathrm{CNN})$ for detecting number plate and same approach for optical character recognition. Besides, the interface of the system is user-friendly and is easier to use. The detection and recognition processes of this system are smooth because of the steps that are used in this system References. This system has the capability of detecting number plate for different conditions like different font size, different font style, hand-written characters, different background color for all Indian number plates. The proposed method is flexible and not 


\section{International Journal of Engineering Applied Sciences and Technology, 2021 \\ Vol. 5, Issue 11, ISSN No. 2455-2143, Pages 314-317 \\ Published Online March 2021 in IJEAST (http://www.ijeast.com)}

sensitive to image variations. The method is resistant for variations of a size of number plate (within a certain interval).

\section{REFERENCE}

[1] Singh Jaskirat, Bhushan Bharat, (2019), "Real time Indian license plate detection using deep Neural networks and optical character recognition using LSTM Tesseract", 10.1109/ICCCIS48478.2019.8974469.

[2] Kaur Kamaljit, Kaur Balpreet, (2013) “Character Recognition of High Security Number Plates Using Morphological Operator”, 10.11591/telkomnika.v13i1.6782.

[3] Reagan L. Galvez, Argel A. Bandala, Elmer P. Dadios, Ryan Rhay P. Vicerra, Jose Martin Z. Maningo, (2018) "Object detection using Convolutional neural network", 10.1109/TENCON.2018.8650517

[4] Meduri Avadesh, Navneet Goyal, (2018), “Optical character recognition for Sanskrit using Convolutional neural network", 10.1109/DAS.2018.50

[5] Ravirathinam Praveen, Patawari Arihant, (2019), "Automatic License Plate Recognition for Indian Roads Using Faster-RCNN", $\underline{10.1109 / \mathrm{ICoAC} 48765.2019 .246853}$

[6] Khichi Monica, Agarwal Chanchal, (2019)" A review on Automatic number plate recognition technology and methods", 10.1109/ISS1.2019.8908014

[7] Bharat V, N. Rani Shobha, (2017), "A font style classification system for English OCR", $\underline{10.1109 / I 2 C 2.2017 .8321962}$

[8] Mehta Mayuri A, Pote Saloni A, (2018), “Text detection from scene videos having blurriness and text of different sizes", 10.1109/PUNECON.2018.8745375

[9] Mehta Honey, Singla Sanjay, Mahajan Aarti, (2016)," Optical Character Recognition (OCR) System for Roman Script \& English Language using Artificial Neural Network (ANN) Classifier", 10.1109/RAINS.2016.7764379

[10] Kashyap Abhishek, B. Suresh, Patil Anukul, Sharma Saksham, Jaiswal Ankit, (2018), "Automatic Number Plate Recognition", 10.1109/ICACCCN.2018.8748287

[11] Shaukat Hayat, She Kun, Zuo Tengtao, Yue Yu, Tianyi Tu, Yantong Du, (2018), “A Deep Learning Framework Using Convolutional Neural Network for Multi-class Object Recognition”, 10.1109/ICIVC.2018.8492777

[12] Thanh-Nga Nguyen, Duc-Dung Nguyen, (2018), “A New Convolutional Architecture for Vietnamese Car Plate Recognition", 10.1109/KSE.2018.8573375 